\title{
Capacitive Coupling Noise in High-Speed VLSI Circuits
}

\author{
Payam Heydari \\ Department of Electrical and Computer Engineering \\ University of California \\ Irvine, CA 92697
}

Massoud Pedram

Department of Electrical Engineering-Systems

University of Southern California

Los Angeles, CA 90089 


\section{Introduction}

Shrinkage of the minimum feature size of the semiconductor devices to $130 \mathrm{~nm}$ and below and increase in the the clock frequency to $3 \mathrm{GHz}$ and above have caused crosstalk noise to become a serious problem in integrated circuits. More precisely, crosstalk noise has

evolved as the key source of performance degradation and signal integrity problems in high speed VLSI designs.

Various techniques have been proposed to evaluate the crosstalk noise in integrated circuits. The most accurate approach is to use a transistor-level circuit simulator. This approach is, however, computationally inefficient, and hence, is not applicable to large circuit structures. For example, our experiments show that simulating a small circuit structure consisting of a collection of 10 coupled lossy transmission lines with HSPICE takes almost three minutes on a $1.5 \mathrm{GHz}$ Intel Pentium-4-based computer system. Since interconnects are modeled as linear time-invariant systems, model reduction techniques [1]-[6] can be utilized to reduce the computational complexity. These model order reduction techniques may be incorporated into the noise analysis and calculation programs to accurately determine the noise behavior of the circuit under study. For example, reference [7] enumerates different types of environmental noise sources that have a major impact on digital VLSI circuit performance. Next it proposes a fast methodology using noise graphs to analyze the noise. The main shortcoming of this work is that it does not accurately model the on-chip interconnects. Reference [8] incorporates a model order reduction technique to efficiently simulate the on-chip interconnects as distributed RC sections. However, it does not present any analytical expression for the crosstalk noise. Moreover, in spite of employing model reduction techniques, this approach cannot completely solve the problem of long computation times associated with this kind of noise analysis. In addition, neither [7] nor [8] provide any insight to the circuit designers as to how to modify the circuit structures in order to reduce or control the crosstalk noise.

It is desirable to use closed-form expressions, instead of simulation tools, to predict the noise effects in a circuit as long as their prediction accuracy is acceptable. This is especially true during the early stages of the design process when one does not afford simulat- 
ing a large number of possible circuit structures and layout solutions. Consequently, a number of researchers have addressed the problem of obtaining simple, closed-form expressions for crosstalk noise in VLSI circuits. Vittal et al. in [9] provide bounds for the crosstalk noise using a lumped RC model. This work, however, ignores the interconnect resistance. Later, the same authors, in [10], make use of geometric considerations to obtain expressions for the peak amplitude and the pulse width of the noise. Knowing the noise pulse width is important because, in general, the noise margin of a gate depends on both the noise peak amplitude and the noise pulse width. Their technique can handle arbitrary input signals. In [11], Devgan proposes a clever technique for finding an upper bound on the crosstalk noise. The author himself mentions that his model exhibits a large error when the signals are fast and the rise and fall times are short. Unfortunately, this latter scenario occurs frequently when practical values of the interconnect parasitics and signal frequencies are used. We have observed that the percentage of the estimated error in such cases can be as much as $60 \%$. In addition, [11] does not predict the noise pulse width.

Kuhlmann et al. in [12] and [14] propose an exact crosstalk noise estimation method for a distributed RC(L) model of the VLSI interconnect. They employ a moment-matching technique and Devgan's metric to effectively reduce the Laplace transform of the coupled interconnect to a low-order rational function. More specifically, for a distributed RC interconnect, a high-order transfer function is first reduced to a third order rational transfer function by using a combination of moment-matching technique and Devgan's metric. The poles of the reduced-order transfer function are then derived to examine the stability of the reduced system. If the reduced system is unstable, a second-order transfer function will be used instead. Authors demonstrate the accuracy of their proposed method by comparing its performance with that of HSPICE. The metric proposed in [12] and [14] is less intuitive in terms of the circuit interpretation, and requires two tree traversals (one for the aggressor net and the other one for the victim net), a diagonal matrix-vector multiplication for the DC component of the Taylor series expansion of the victim voltage in the Laplacedomain, two additional tree traversals per moment, and two diagonal matrix-vector multiplication for high moments of the victim and the aggressor voltages. The tree traversal and the multiplication are of the order of $\mathrm{O}(n)$ for $n$ nodes. In addition, for every run of the cir- 
cuit simulator on the system, roots of the reduced third-order model should be obtained for the stability check. This operation is also of the order of $\mathrm{O}(n)$. The number of tree traversals and matrix multiplications to generate the third-order model are six times and twenty times more than those in the Devgan's metric, respectively [14]. [13] proposed a conventional lumped 2-p RC circuit for the victim net, while postulating that the slew rate at the coupling location is obtained by simple slew-rate calculation from the aggressor driver, which is not accurately correct for long parallel wires. The reason is that the slew-rate calculation for the aggressor net must account for the distributed nature of the coupling between the adjacent lines. Although a lumped 2- $\pi$ model can help us derive closed-form analytical models for the noise attributes, the results lead to large and unaccetable errors for long aggressor and victim nets. Using this model, the authors calculated the noise peak and pulse width, and defined the amplitude pulse width product. Several experiments in this paper have shown that the noise peak amplitude will have a more contribution to the circuit failure than the noise pulse width. Therefore, a new definition is needed to emphasize the bigger impact of the noise peak amplitude. Takahashi et al. in [15] proposes a 2- $\pi$ equivalent circuit to estimate crosstalk noise of partially coupled RC trees. This paper assumes the aggressor waveform to be an exponential function, which then yields a more accurate estimate than that case that either a step input or a saturated ramp input are assumed. Unfortunately, the proposed analytical model for the interconnect is a $2-\pi \mathrm{RC}$ network, which cannot capture the distributed nature of a long RC interconnect. This is the major shortcoming of the work by Takahashi et al. [16] has proposed a fast aggressor and tree reductions to estimate the crosstalk. Similar to [13] and [15], [16] employs doublepole approach for the crosstalk noise estimation.

In this paper, a new crosstalk noise metric is proposed, which is capable of predicting the noise amplitude and the noise pulse width of an $\mathrm{RC}$ interconnect as well as an overdamped RLC interconnect very efficiently. This paper is based on the work originally proposed in [17]. Several experiments reveal that the proposed metric, on average, predicts the peak crosstalk noise with the same or higher level of accuracy compared to the secondorder reduced model proposed in [14]. Unlike [14], the proposed noise metric gives a rather simple analytical model of the crosstalk noise, which is efficient and sufficiently 
accurate to be effectively incorporated in state-of-the-art noise calculators. The proposed noise metric has a closed form expression that clearly highlights the dependency of the noise on the aggressor and victim line circuit parameters as well as on the input signal rise/ fall times.

This paper is organized as follows. In Section 2, the interconnect coupling phenomenon is reviewed and, through experimental results, it is shown that the inductive coupling on chip is negligible for local wiring clocked at a target frequency of $1 \mathrm{GHz}$. This result justifies the focus of this paper, which is on the capacitive crosstalk effect. After a brief description of Devgan's metric in Section 2.1, we introduce our new noise metric in Section 2.2. We next compare our metric with the analytical models proposed by Vittal [10], Devgan [11], and Kuhlmann [12], [14] through a series of detailed simulation experiments. Finally, Section 3 provides the conclusion of this paper.

\section{Capacitive Coupling}

The electromagnetic coupling of a signal from one conductor to another, which is called crosstalk, can be induced through two coupling mechanisms: capacitive and inductive.

All signal conductors exhibit some interwire capacitances among themselves. When the conductors are placed sufficiently close to each other, the capacitance becomes large enough to couple significant energy from one conductor, called an aggressor or active line, to another conductor, called a victim or passive line. Because, with each new process technology, the thickness (height) of the wires is not scaled down as aggressively as the width of the wires, and because the wires are packed increasingly closer to each other, the ratio of the coupling capacitance to the total capacitance (includes area and fringe capacitances) increases, and therefore, the capacitive coupling noise increases. Fig. 1 depicts a highly simplified analysis (neglecting the resistive loss and the magnetic coupling of the interconnect lines) of the essential attributes of the crosstalk noise. In this figure, coupled noise on line 2 results from a transition on line 1. In this simplified circuit model, the crosstalk voltage is obtained from a capacitive voltage division relationship as follows:

$$
V_{\text {xtalk }}(t)=\left(\frac{C_{c}}{C_{c}+C_{\text {tot } 2}}\right) V_{D D} e^{-t / \tau} u(t) \text { where } \quad \tau=r_{D S, 12}\left(C_{c}+C_{\text {tot } 2}\right)
$$


where $u(t)$ represents the unit-step function, and $r_{D S, 12}$ is the on resistance of the NMOS device of the victim line driver.

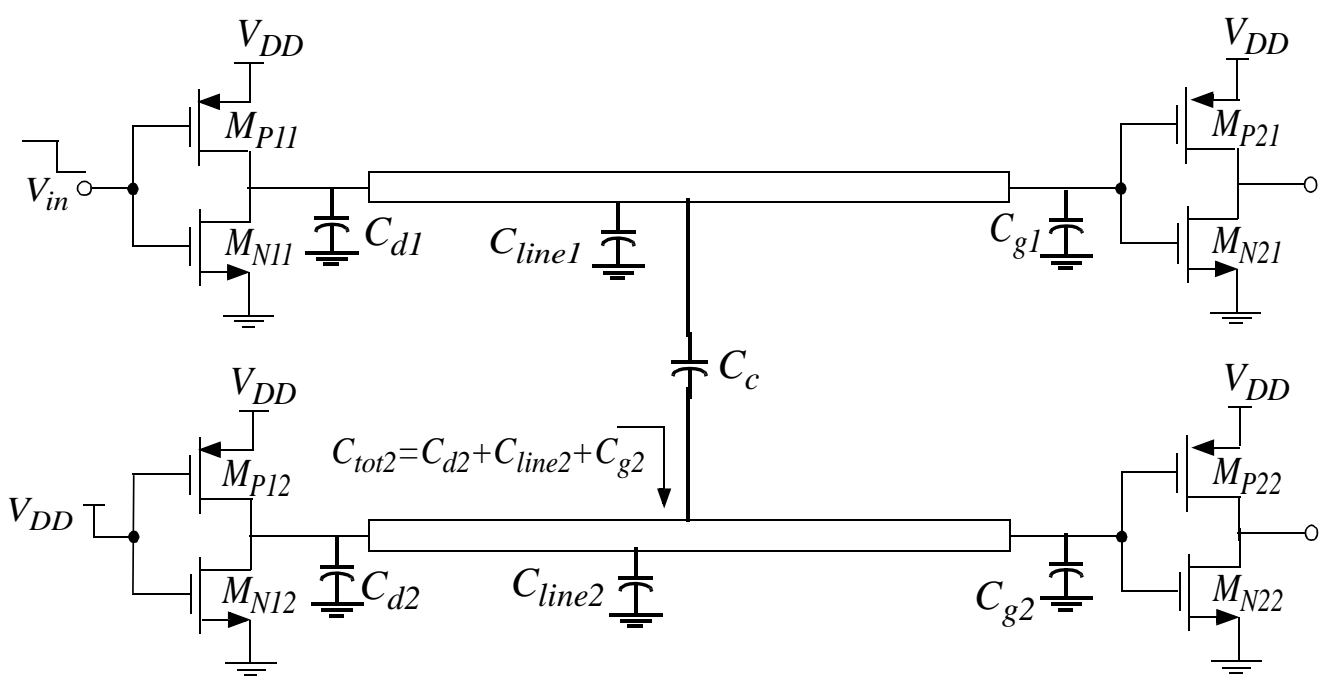

Fig. 1. A simplified circuit model of two capacitively coupled transmission lines.

High-speed digital circuits often employ dynamic logic families (e.g., Domino or True Single-Phase Clocking) due to their higher switching speeds compared to the static logic family. Dynamic circuits are, however, more susceptible to crosstalk noise compared to the static logic because during some phase of the clock, the logic value is only stored on a floating capacitor. An induced noise that changes the logic value on this floating capacitor can cause the circuit to produce an incorrect result. Furthermore, as the circuit speed increases and the signal transient times decrease, the effects of on-chip crosstalk noise becomes more pronounced. Fig. 2 shows $N$ neighboring wires. High frequency operation of VLSI circuits causes on-chip wires to exhibit transmission line effects, and hence, electrical and magnetic couplings start to take place between pairs of wires. These electric and magnetic couplings reshape the signal waveforms and may induce delay in the signals traveling through the lines, thus causing the circuit to possibly violate its timing constraints. 


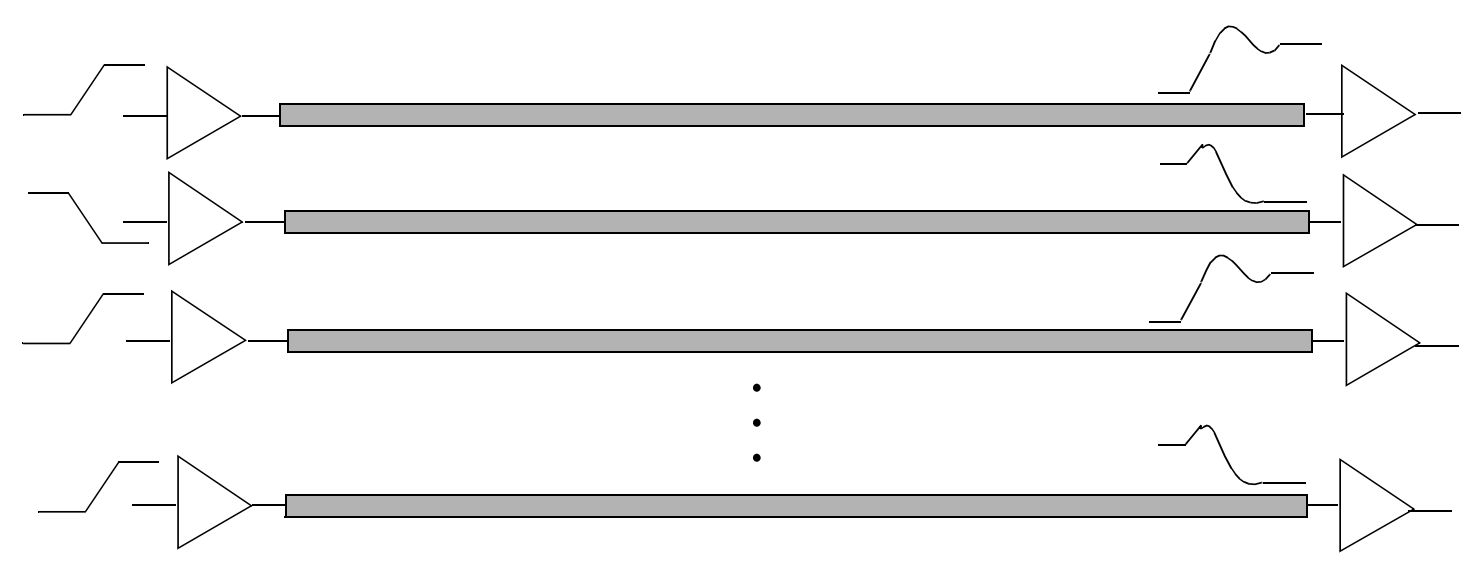

Fig. 2. Circuit schematic of $N$ on-chip interconnects.

Our goal is to develop a circuit model for the capacitive coupling between on-chip coupled interconnects and then use this model to derive a closed-form expression for the crosstalk noise. We start our analysis by reviewing Devgan's metric and its drawbacks in estimating the crosstalk noise in RC circuits. For a more comprehensive explanation of this metric, please refer to [11].

\subsection{Devgan's metric for crosstalk noise estimation}

Consider two capacitively coupled RC networks as shown in Fig. 3.
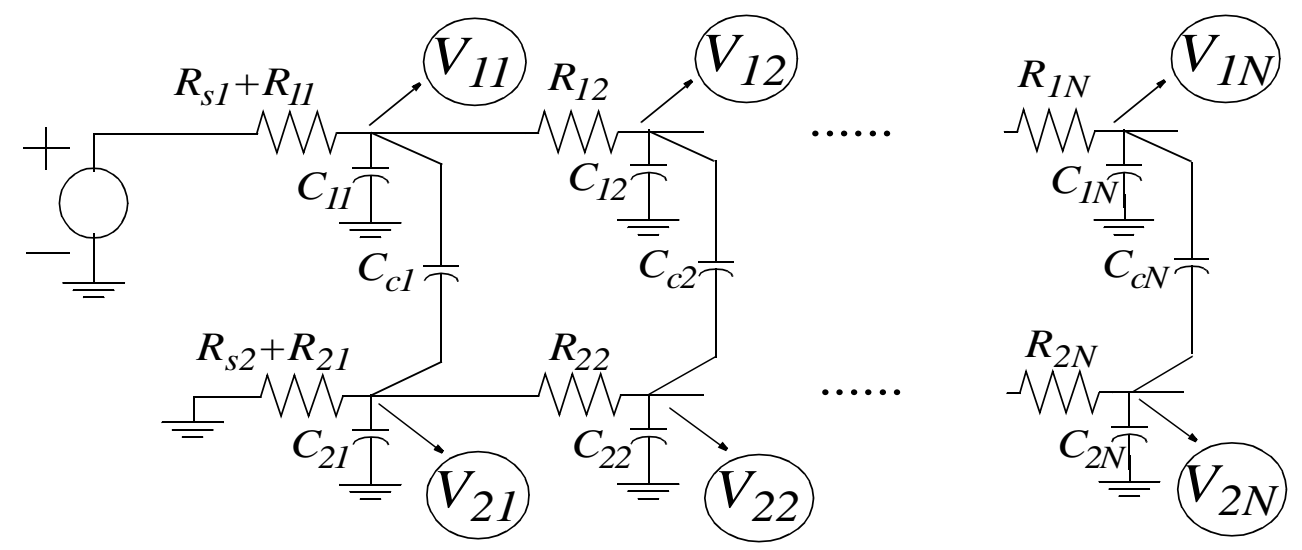

Fig. 3. Circuit schematic of capacitively coupled aggressor and victim nets.

The RC ladder network representing the aggressor net is driven by a flattened ramp voltage whereas the RC ladder network representing the victim net is quiet. For this cir- 
cuit, the node voltage vector at the victim net, $\mathbf{V}_{2} \in \mathfrak{R}^{N \times 1}$, is related to the voltage vector at the aggressor net, $\mathbf{V}_{1} \in \mathfrak{R}^{N \times 1}$, through the following equation:

$$
\left[\left(s \mathbf{C}_{2}-\mathbf{A}_{22}\right)-s \mathbf{C}_{c}\left(s \mathbf{C}_{1}-\mathbf{A}_{11}\right)^{-1}{ }_{s} \mathbf{C}_{c}\right] \mathbf{V}_{2}={ }_{-s} \mathbf{C}_{c}\left(s \mathbf{C}_{1}-\mathbf{A}_{11}\right)^{-1} \mathbf{B}_{1} \mathbf{V}_{s}
$$

where $\mathbf{C}_{i}=\operatorname{diag}\left(C_{i j}+C_{c j}\right)$ for $i=1,2$ and $j=1,2, \ldots, N$ and $\mathbf{C}_{c}=\operatorname{diag}\left(-C_{c j}\right)$ for $j=1,2, \ldots, N . \mathbf{A}_{11}$ and $\mathbf{A}_{22}$ represent the equivalent node resistance matrices of the aggressor net and the victim net, respectively. The steady-state values of node voltages at the victim net is calculated as:

$$
\mathbf{V}_{2, s s}=-\mathbf{A}_{22}^{-1} \mathbf{C}_{c} \mathbf{A}_{11}^{-1} \mathbf{B}_{1} \frac{V_{D D}}{t_{r}}
$$

where $t_{r}$ is the rise-time of the input signal of the aggressor line driver. For simplicity, it is assumed that the rise and fall times are equal. Note that this result is valid only if the driving voltages of the interconnects are infinite ramps. This is a critical assumption that seriously and adversely affects the accuracy of capacitive crosstalk estimation. In practice, the actual driving voltages of the interconnects are saturated ramp inputs rather than infinite ramps. This means that the node voltages at the victim net reach their peak value approximately at $t=t_{r}$. This peak value is obviously different from the steady-state value under the infinite ramp input, and the error between these two values can be quite large if the rise-time of the input is fast.

To better understand the shortcoming of this approach, consider two second-order RC circuits with two floating capacitances connecting the corresponding nodes of these two circuits as depicted in Fig. 4. $R_{s 1}$ and $R_{s 2}$ represent the resistances of the input source. In reality, they represent the on-resistances of line drivers. The output impedance of line drivers can be modeled as another RC section that is connected to the distributed RC interconnect. Consider typical values of these parasitics, i.e., assume that $C_{1}=60 \mathrm{fF}, C_{2}=$ $120 \mathrm{fF}, R_{2}=50 \Omega, R_{1}=20 \Omega, C_{c}=180 \mathrm{fF}, R_{s 1}=100 \Omega, R_{s 2}=150 \Omega$, and $t_{r}=0.1 \mathrm{~ns}$. 


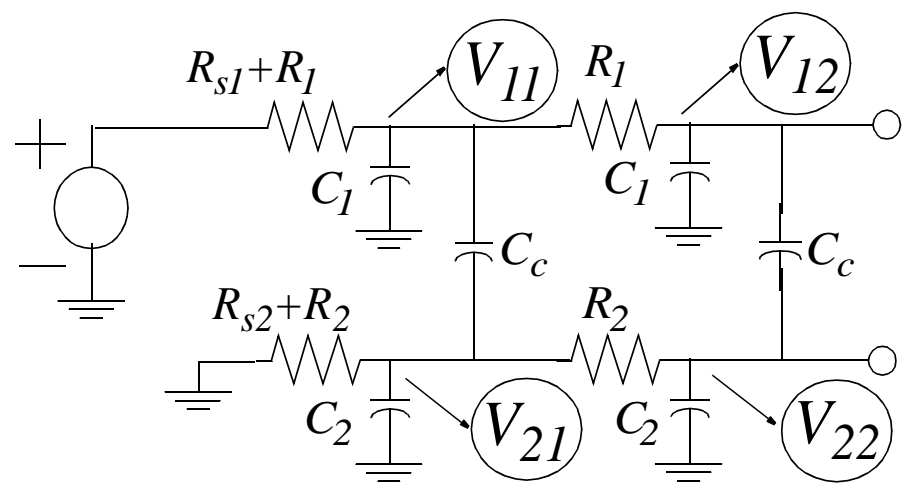

F20 ig. 4. A pair of capacitively coupled second-order RC circuits.

From HSPICE simulation, the reported peak value of voltage $V_{22}$ at the far-end of the victim line is $0.416 \mathrm{~V}$. Devgan's metric for the two coupled RC sections yields the following equations:

$$
\begin{gathered}
V_{21, s s}=2\left(R_{2}+R_{s_{2}}\right) C_{c} \frac{V_{D D}}{t_{r}} \\
V_{22, s s}=\left(3 R_{2}+2 R_{s_{2}}\right) C_{c} \frac{V_{D D}}{t_{r}}
\end{gathered}
$$

Using Eq. (4), $V_{22, s s}$ is $1.053 \mathrm{~V}$. The estimated error is $153 \%$ verifying a well-known observation established by earlier published works that this metric can be inaccurate for deep sub-micron technologies [14].

Because the input signal rise-time is small, the crosstalk waveform rolls down quickly, and consequently, the error becomes unacceptably large ( $c f$. Fig. 5). Notice that for cases where the input signal rise-time is large compared to the interconnect delays, Devgan's metric accurately predicts the peak value. Unfortunately, cases in which the estimations are accurate (i.e., the slow slew rates for the pulses), are not the most important ones from a circuit performance viewpoint. The reason is that the peak value of the crosstalk is inversely proportional to the input rise-time. For slow slew waveforms, the crosstalk also has a small peak value, and therefore, it has little impact on the circuit delay and the timing failure rate. 


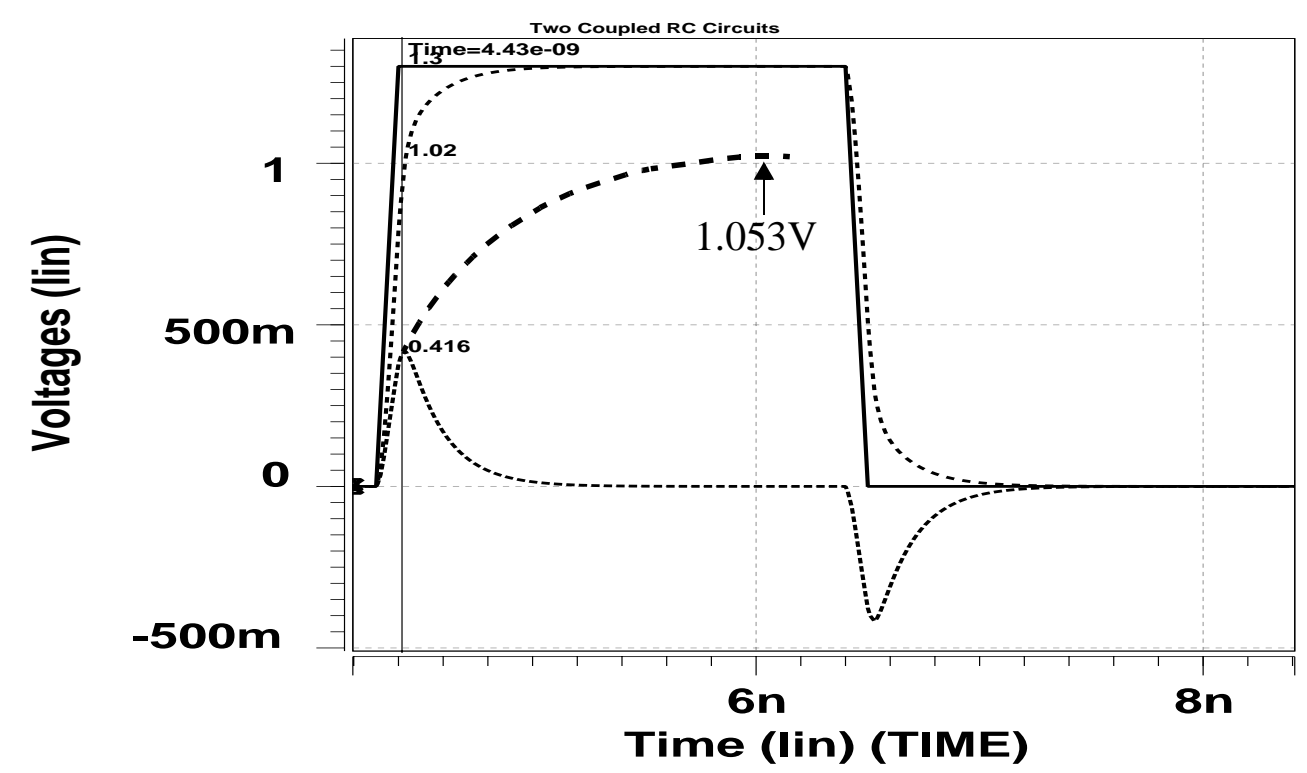

Fig. 5. The output voltage and the crosstalk of two coupled second-order RC circuits. $C_{1}=60 \mathrm{fF}, C_{2}=120 \mathrm{fF}, R_{2}=50 \Omega, R_{1}=20 \Omega, C_{c}=180 \mathrm{fF}, R_{s 1}=100 \Omega, R_{s 2}=150 \Omega$, and $t_{r}$ $=0.1 \mathrm{~ns}$.

In the next section, we derive a new, more accurate noise metric, and compare our results with Devgan's results and with HSPICE simulations.

\subsection{A new metric for crosstalk noise estimation}

Close examination of the HSPICE results shown in Fig. 5 reveals a major source of inaccuracy in Devgan's metric. The large error in this example comes from the fact that the time constants of the exponentially rising portions of the victim node voltages, $V_{2 j}$ for $j=$ $1,2, \ldots, N$, in the circuit of Fig. 5 are comparable to (or larger than) the input rise time. The actual peak value of the crosstalk occurs approximately at $t=t_{r}$ (or $t_{f}$, the fall-time, whatever the case may be). In fact, other works (e.g., [13]) proved theoretically that the peak noise is at $t_{r}$, under one-pole or two-pole approximation. For a flattened ramp input, it is easily seen that $t_{r(f)}$ sets a lower bound on the time instance at which the peak value of the crosstalk occurs. This is because, for an infinite ramp input, the voltage waveforms 
at the victim line nodes monotonically increase toward their steady state values as predicted by [11]. As the current drive capability of the line drivers decreases or as the driver sizes of the aggressor and victim lines become very different from one another, the peak value of the crosstalk may occur further away from $t=t_{r(f)}$. In contrast, as will be demonstrated through a number of circuit simulations later in this section, our proposed metric produces an accurate noise peak value and noise pulse width for all possible scenarios with regard to the victim and aggressor line drivers.

To compute the noise peak value, we observe that the capacitive crosstalk noise at every node of the victim net is a rising exponential function during the time interval that the input signal of the aggressor line driver is rising. The actual peak value of the crosstalk noise at each node of the victim net is in fact the value of the corresponding rising exponential function at $t=t_{r(f)}$. Recall that the steady-state value of this exponential function is determined by Devgan's metric.

$$
\mathbf{V}_{2, \text { max }}=\mathbf{V}_{2, s s}\left(\mathbf{I}-\mathbf{e x p}\left(\operatorname{diag}\left(-\frac{t_{r(f)}}{\tau_{d_{j}}}\right)\right)\right) \quad \text { for } j=1,2, \ldots, N
$$

where $\operatorname{diag}(\mathbf{x})$ represents a diagonal matrix with all diagonal entries set to $\mathbf{x} . \tau_{d_{j}}$ is the time constant of the $j$-th node voltage in the victim net, and $\mathbf{V}_{2, s s}$ is the vector of steady state values of the crosstalk noise voltages at the victim nodes as calculated by Devgan's metric. Each node in the victim net sees two capacitances: a grounded area capacitance, $C_{2 j}$, and a floating coupling capacitance, $C_{c j}$. The time constant at each victim node is thus equal to the summation of individual time constants due to each of these two capacitances. Similar to the open-circuit time-constant method that is employed for estimating the bandwidth of high-frequency amplifiers [18], the time-constant due to each capacitance is obtained by calculating the equivalent resistance seen across each capacitance with all the other capacitances open-circuited. Therefore, to accurately estimate the time constants due to capacitances $C_{2 j}$ and $C_{c j}$, we first construct an equivalent circuit consisting of $C_{2 j}, C_{c j}$, and the equivalent resistances seen across these two capacitances and replace all of the other capacitances with open circuit connections. This circuit model is shown in Fig. 6. 


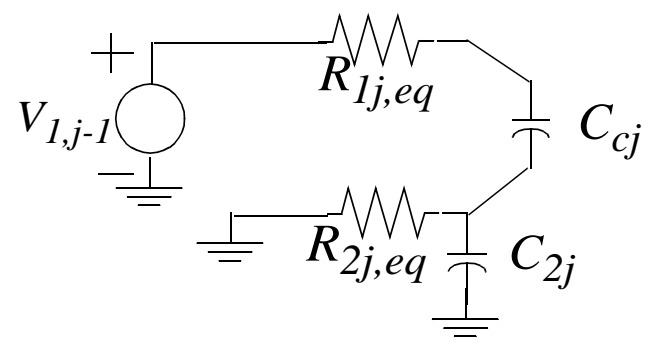

$$
\begin{aligned}
& R_{2 j, e q}=\sum_{\substack{i=1 \\
j}}^{j} R_{2 i} \\
& R_{1 j, e q}=\sum_{i=1} R_{1 i}
\end{aligned}
$$

Fig. 6. The equivalent circuit for computing the time constant of the $j$-th node of the victim net.

The characteristic polynomial of this second-order transfer function is:

$$
\Lambda_{j}(s)=R_{1 j, e q} R_{2 j, e q} C_{2 j} C_{c j} s^{2}+\overbrace{\left[\left(R_{1 j, e q}+R_{2 j, e q}\right) C_{c j}+R_{2 j, e q} C_{2 j}\right] s+1}^{\tau_{v_{j}}}
$$

The time constant of this second-order circuit, which is roughly the inverse of the 3-dB bandwidth of its system transfer function, is equal to the coefficient of the first-order term, denoted by $\tau_{v_{j}}$. In fact, $\tau_{d_{j}}$ must contain this coefficient as a part of its expression. Notice that the input voltage source must be a unit-step function for $\tau_{v_{j}}$ to properly represent the time constant at the $j$-th node of the victim net. This is obviously not the case for the distributed coupled RC circuits. The input voltage to the $j$-th node of the aggressor experiences an RC delay due to the RC path from the input to the $j$-th node of the aggressor as is shown in Fig. 7. The RC delay cannot be computed using the Elmore delay formula because there is a signal path from the input node to each victim node $V_{2 j}$ through the floating capacitance $C_{c j}$ that connects this victim node to the corresponding aggressor node $V_{l j}$. This RC delay also ought to be accounted for in the delay calculation of Fig. 7. The overall RC delay is thus computed differently from the Elmore delay. 


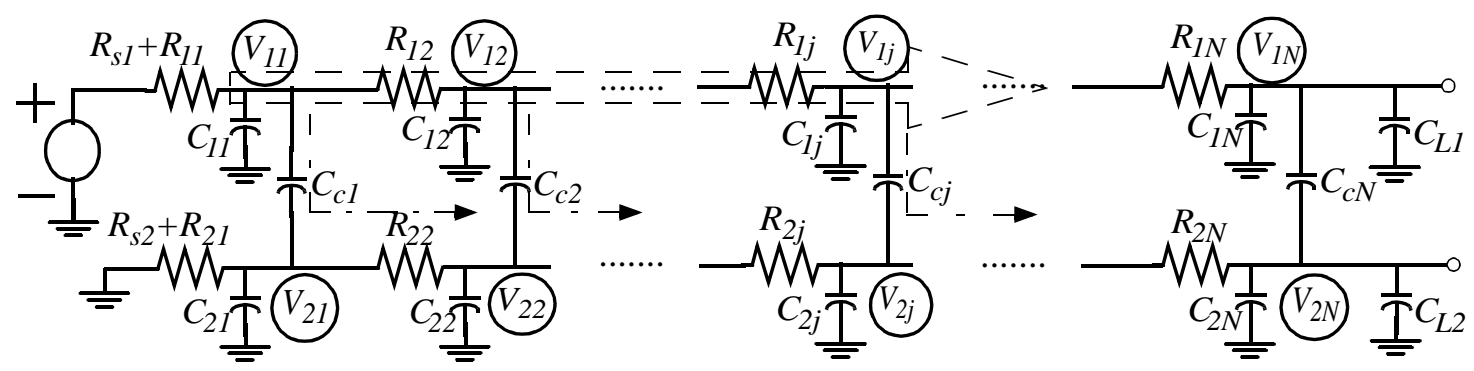

Fig. 7. The RC signal paths through the aggressor line and floating capacitances

Furthermore, for RC circuits with orders greater than one, the initial slopes of the step and ramp responses are zero. This zero initial slope leads to an increase in the circuit delay. Fig. 8 indicates all these delay effects on the crosstalk noise waveform for a flattened ramp input as well as a step input.

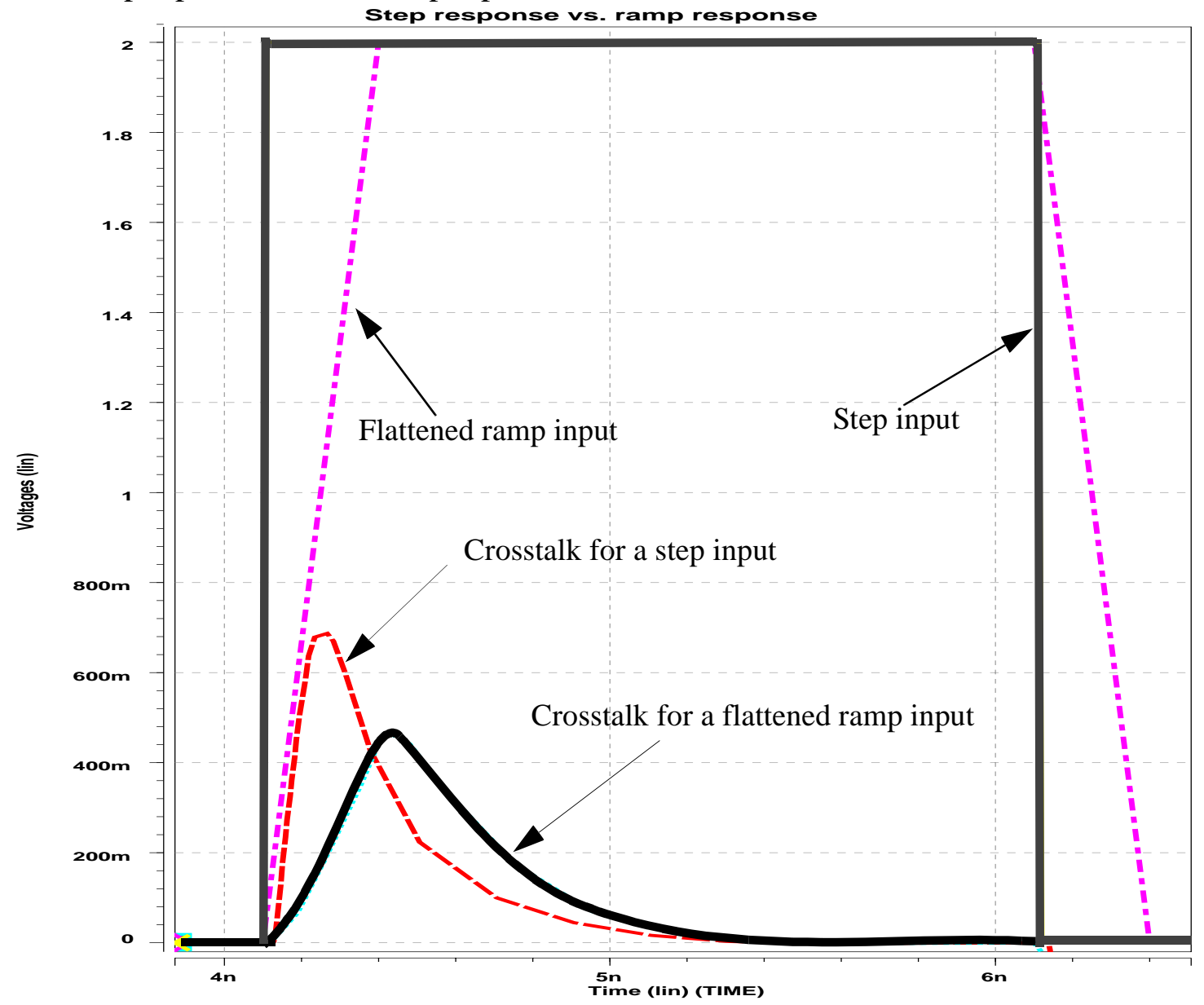

Fig. 8. Effects of zero initial slope and RC delay on the crosstalk. 
Consequently, the time constant of the $j$-th node in the victim net consists of two additive terms $\tau_{v_{j}}$ and $\tau_{a_{j}}$, with $\tau_{v_{j}}$ (given by Eq. (6)) represents the time constant of the $j$-th node in the victim net under a unit-step input excitation, and $\tau_{a_{j}}$ represents the propagation delay of the signal coming from other paths established by the floating capacitances $C_{c k}(k=1, \ldots, j-1)$ as illustrated above. According to Fig. 7, $\tau_{a_{j}}$ includes the signal delays of all additional signal paths through the coupling capacitances $C_{c k}(k=1, \ldots, j-1)$ toward the $j$-th node in the victim net. The overall delay from the aggressor input source to the $j$-th node in the victim net is:

$$
\tau_{d_{j}}=\zeta \cdot\left[\left(R_{1 j, e q}+R_{2 j, e q}\right) C_{c j}+R_{2 j, e q} C_{2 j}+\tau_{a_{j}}\right] \quad \text { for } j=1,2, \ldots, N
$$

where $\tau_{a_{j}}$ is:

$$
\begin{aligned}
\tau_{a_{j}}=R_{1 j, e q}\left(C_{c j}+C_{1 j}\right)+\sum_{k=1}^{j-1}\left[R_{1 k, e q}\left(C_{c k}+C_{1 k}\right)+R_{2 k, e q}\left(C_{2 k}+C_{c k}\right)\right] & \\
& \text { for } j=1,2, \ldots, N
\end{aligned}
$$

and $\zeta$ is a constant factor for the delay increase due to the nonzero, yet finite, input slope. Its value is in the range $[1.00,1.02]$. Throughout our analysis, we use $\zeta=1.01$. Combining equations (7) and (8) yields the following expression for $\tau_{d_{j}}$ :

$$
\begin{aligned}
& \tau_{d_{j}}=\zeta \cdot\left[R_{1 j, e q} C_{c j}+\sum_{k=1}^{j}\left[R_{1 k, e q}\left(C_{c k}+C_{1 k}\right)+R_{2 k, e q}\left(C_{2 k}+C_{c k}\right)\right]\right] \\
& \text { for } j=1,2, \ldots, N
\end{aligned}
$$

The peak amplitude of the crosstalk is easily obtained from equation (5) with the expression for $\tau_{d_{j}}$ given by equation (9).

As a special case, we first concentrate on the circuit of Fig. 4 in which two secondorder RC circuits are capacitively coupled. The peak voltage value of the node $V_{22}$ is calculated using three different approaches; HSPICE simulation, Devgan's metric, and our proposed analytical model. Applying equations (5) and (9) to the circuit of Fig. 4 yields 
the following closed-form expressions for the peak values of the nodes, $V_{21}$ and $V_{22}$ :

$$
V_{21, \text { max }}=V_{21, s s}\left(1-\exp \left(-\frac{t_{r}}{\tau_{d_{1}}}\right)\right)
$$

where $\quad \tau_{d_{1}}=1.01\left[\left(R_{1}+R_{s 1}\right)\left(2 C_{c}+C_{1}\right)+\left(R_{2}+R_{s 2}\right)\left(C_{c}+C_{2}\right)\right]$

$$
V_{22, \text { max }}=V_{21, s s}\left(1-\exp \left(-\frac{t_{r}}{\tau_{d_{2}}}\right)\right)
$$

where $\tau_{d_{2}}=1.01\left[\left(3 R_{1}+2 R_{s 1}\right)\left(C_{c}+C_{1}\right)+\left(3 R_{2}+2 R_{s 2}\right)\left(C_{2}+C_{c}\right)+\left(2 R_{1}+R_{s 1}\right) C_{c}\right]$

To verify the accuracy of our approach on multistage RC networks in comparison to other expressions proposed in [10], [13] and [14], we perform a number of experiments on a two-line structure in a 130nm CMOS technology. In state-of-the-art CMOS technologies the coupling capacitance accounts for approximately $70-95 \%$ of the total node capacitances, which makes the coupling noise analysis even more important. In our implementation of the algorithm presented in [14], the second-order reduced transfer function is utilized in order to avoid a potential stability problem. The coupled lengths of the adjacent interconnects are varied from $200 \mu \mathrm{m}$ to $8 \mathrm{~mm}$. The supply voltage is $V_{D D}=1.3 \mathrm{~V}$. Results are reported for a range of rise-times varying between 30ps and 200ps, and for different victim and aggressor driver resistances varying between $20 \Omega$ and $5 \mathrm{k} \Omega$. Table 1 contains the result of these comparisons. Of particular interest is the situation where the driver strengths of the aggressor and victim lines are vastly different. For instance, this circuit configuration is encountered when a global signal line is in the close vicinity of a local signal line. The last five experiments in Table 1 are devoted to this particular configuration. The mean and maximum error values are reported in Table 2. These tables testify to the higher accuracy of our approach compared to these other approaches. More precisely, our proposed analytical model results in an average estimation error of only $5.82 \%$ which is better than the $6.81 \%$ average estimation error resulting from the method of reference [14] when a second-order reduced transfer function is employed. Interestingly, the proposed noise metric exhibits a better accuracy compared to [14] when the driver sizes of 
Table 1: The results of simulations on the two capacitively coupled transmission lines using star-HSPICE, comparing methods of references [10], [13], [14] and our proposed metric using a 130nm technology and $V_{D D}=1.3 \mathrm{~V}$. The aggressor line input is a flattened ramp signal

\begin{tabular}{|c|c|c|c|c|c|c|c|c|c|c|c|c|c|c|c|}
\hline $\begin{array}{l}c_{1} \\
(p F / m)\end{array}$ & $\begin{array}{l}\mathbf{r}_{1} \\
(\mathbf{k} \Omega / \mathbf{m})\end{array}$ & $\begin{array}{l}\mathbf{r}_{2} \\
(\mathbf{k} \Omega / \mathbf{m})\end{array}$ & $\begin{array}{l}\mathrm{c}_{2} \\
(\mathrm{pF} / \mathrm{m})\end{array}$ & $\begin{array}{l}\mathrm{c}_{\mathrm{c}} \\
(\mathrm{pF} / \mathrm{m})\end{array}$ & $\mathbf{R}_{\mathrm{s} 1}$ & $\mathbf{R}_{\mathrm{s} 2}$ & $\begin{array}{l}\mathrm{C}_{\text {out1 }} \\
(\mathrm{pF})\end{array}$ & $\begin{array}{l}\mathrm{C}_{\text {out2 }} \\
(\mathrm{pF})\end{array}$ & $\begin{array}{l}\mathbf{t}_{\mathbf{r}} \\
\text { nsec }\end{array}$ & $\begin{array}{l}\mathbf{L} \\
(\mathbf{m m})\end{array}$ & $\begin{array}{l}\text { HSPICE } \\
\text { volts }\end{array}$ & $\begin{array}{l}\text { Pan } \\
\text { volts }\end{array}$ & $\begin{array}{l}\text { Vittal } \\
\text { volts }\end{array}$ & $\begin{array}{l}\text { Kuhlmann } \\
\text { volts }\end{array}$ & $\begin{array}{l}\text { Ours } \\
\text { volts }\end{array}$ \\
\hline$\overline{60}$ & 11.47 & $\overline{10.2}$ & 64 & 100 & $\overline{1 k}$ & $2 \mathbf{2 k}$ & 0.09 & $\begin{array}{l}\mathbf{0 . 0 8} \\
\end{array}$ & 0.05 & 0.2 & 0.113 & 0.137 & 0.1396 & 0.119 & 0.1271 \\
\hline 72 & 9.55 & 9.55 & 72 & 150 & 527 & 527 & 0.2 & 0.1 & 0.04 & 0.7 & 0.168 & 0.201 & 0.2111 & 0.192 & 0.181 \\
\hline 83 & 8.2 & 7.0 & 90 & 160 & 1013 & 920 & 0.3 & 0.1 & $\mathbf{0 . 0 3}$ & 0.8 & 0.143 & 0.165 & 0.1937 & 0.152 & 0.16 \\
\hline 92 & 9.3 & 10 & 80 & 170 & 270 & 400 & 0.06 & 0.2 & 0.06 & 2.5 & 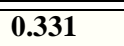 & 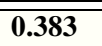 & $\overline{0.401}$ & $\overline{0.352}$ & $\overline{0.361}$ \\
\hline 101 & 12 & 12 & 101 & 150 & 140 & 150 & 0.1 & 0.2 & 0.08 & 1.2 & 0.189 & 0.221 & $\overline{0.2096}$ & 0.202 & 0.1901 \\
\hline 120 & 10 & 10 & 120 & 132 & $2 k$ & $4 k$ & 0.06 & 0.07 & 0.1 & 1.1 & 0.255 & 0.286 & 0.3573 & 0.268 & 0.285 \\
\hline 108 & 15 & 15 & 108 & 200 & 20 & 30 & 0.2 & 0.1 & 0.15 & 1.3 & 0.0901 & 0.0972 & 0.1234 & 0.0905 & 0.089 \\
\hline 130 & 13 & 20 & 100 & 220 & 670 & 720 & 0.3 & 0.05 & 0.09 & 1.6 & 0.26 & 0.278 & 0.324 & 0.2782 & 0.267 \\
\hline 140 & 17 & 17 & 100 & 200 & 350 & 350 & 0.3 & 0.2 & 0.12 & 2 & 0.222 & 0.261 & 0.271 & (0.239 & 0.221 \\
\hline 140 & 17 & 17 & 100 & 200 & 20 & 30 & $\begin{array}{l}0.07 \\
\end{array}$ & 0.08 & 0.06 & 2 & 0.25 & 0.263 & 0.26 & 0.273 & 0.25 \\
\hline 90 & 11 & 11 & 90 & 120 & 160 & 160 & 0.3 & 0.2 & 0.08 & 4 & 0.219 & 0.254 & 0.266 & 0.238 & 0.232 \\
\hline 85 & 12 & 8.5 & 75 & 140 & 190 & 100 & 0.8 & 0.2 & 0.1 & 5 & 0.144 & 0.156 & $\overline{0.1847}$ & 0.157 & 0.153 \\
\hline 65 & 13 & 7 & 120 & 170 & 600 & 80 & 0.08 & 0.5 & 0.05 & 6 & 0.075 & 0.089 & 0.1 & 0.078 & 0.082 \\
\hline 110 & 6.5 & 7 & 90 & 180 & 80 & 75 & 0.6 & 0.7 & 0.08 & 7 & 0.238 & 0.261 & 0.305 & 0.264 & 0.259 \\
\hline 100 & 8 & 14 & 40 & 110 & 200 & $1 k$ & 0.8 & 0.05 & 0.06 & 8 & $\overline{0.465}$ & $\overline{0.512}$ & $\overline{0.6365}$ & $\overline{0.489}$ & 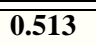 \\
\hline 92 & 13 & 7 & 170 & 260 & $1.5 \mathrm{k}$ & 50 & 0.08 & 0.3 & 0.08 & 0.8 & $\mathbf{0 . 0 2 1 8}$ & 0.0231 & 0.0232 & 0.0224 & $\begin{array}{c}\mathbf{0 . 0 2 2 9} \\
\end{array}$ \\
\hline 110 & 8 & 4 & 200 & 300 & $1.2 \mathrm{k}$ & 25 & 0.1 & 0.65 & 0.1 & 3 & 0.0185 & 0.0207 & 0.0212 & 0.02 & 0.0192 \\
\hline 70 & 10 & 1.82 & 20 & 95 & 20 & $3 \mathbf{k}$ & 0.2 & 0.07 & 0.2 & 1.1 & $\overline{0.573}$ & $\overline{0.584}$ & $\overline{0.584}$ & $\overline{0.597}$ & $\overline{0.596}$ \\
\hline 100 & 7 & 16 & 14 & 100 & 37 & $5 k$ & 0.8 & 0.1 & 0.1 & 2 & 0.647 & 0.689 & $\mathbf{0 . 7 2 7 4}$ & 0.688 & 0.693 \\
\hline 97 & 9 & 15 & 30 & 120 & 26 & $2 k$ & 0.3 & 0.06 & 0.07 & 1.2 & 0.675 & 0.712 & 0.7062 & 0.724 & 0.6862 \\
\hline
\end{tabular}

Table 2: Percentage Error comparison for methods of references [10], [13], [14] and our proposed metric

\begin{tabular}{|c|c|c|c|c|}
\hline & $\begin{array}{c}\text { \% Error } \\
\text { Pan's }\end{array}$ & $\begin{array}{l}\text { \% Error } \\
\text { Vittal's }\end{array}$ & $\begin{array}{c}\text { \%Error } \\
\text { Kuhlmann }\end{array}$ & $\begin{array}{c}\% \text { Error } \\
\text { Ours }\end{array}$ \\
\hline & 21.2 & $\overline{23.54}$ & 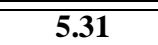 & $\overline{12.48}$ \\
\hline & 19.64 & 35.45 & 14.29 & 7.74 \\
\hline & 15.4 & 26.6 & 6.29 & 11.89 \\
\hline & 15.7 & 21.15 & 6.34 & 9 \\
\hline & 16.9 & 10.9 & 6.88 & 0.5 \\
\hline & 12.16 & 40.12 & 5.1 & 11.76 \\
\hline & 7.88 & 36.9 & 0.4 & 1.2 \\
\hline & 6.9 & 24.62 & 8.46 & 2.69 \\
\hline & 17.6 & 22.07 & 7.66 & 0.4 \\
\hline & 5.2 & 4.0 & 9.2 & 0 \\
\hline & 15.98 & 21.46 & 8.68 & 5.94 \\
\hline & 8.33 & 28.26 & 9.03 & 6.25 \\
\hline & 18.67 & 33.3 & 4 & 9.33 \\
\hline & 9.66 & 28.15 & 10.92 & 8.82 \\
\hline & 10.1 & 36.88 & 5.16 & 10.32 \\
\hline & 5.96 & 6.4 & 2.75 & 0.4 \\
\hline & 11.89 & 14.6 & 8.11 & 3.78 \\
\hline & 1.92 & 1.92 & 4.2 & 4.1 \\
\hline & 7.73 & 12.43 & 6.34 & 7.11 \\
\hline & 5.48 & 4.62 & 7.26 & 2.67 \\
\hline Average & 11.72 & 21.67 & 6.81 & 5.82 \\
\hline Maximum percentage error & 19.64 & 40.12 & 14.29 & 12.48 \\
\hline
\end{tabular}


aggressor and victim lines are hugely different (i.e., the last five rows of Tables 1 and 2). We expect that the method proposed in [14] gives rise to a higher accuracy once the thirdorder reduced transfer function is used. However, this increased accuracy comes at the expense of higher computational complexity due to the stability evaluation of the reduced system. Notice that the metric proposed in [12] and [14] involves multiple tree-traversals, diagonal matrix-vector multiplications, each of which is of order $O(n)$ where $n$ is the number of segment points, in order to compute each moment of each victim net node. From these moments, the noise waveforms at all victim nodes are subsequently calculated by solving a linear system of equations. In contrast, our metric presents closed-form expressions for noise waveforms at all victim nodes (including the far-end termination) with a computational complexity of $O(n)$. The approach proposed in [13] gives rise to a slightly better accuracy for short aggressor nets, as also demonstrated in Table 1.

The run-times of the proposed noise metric with that of [14] is compared in Table 3. In Table 3, the peak value of the far-end crosstalk in a pair of geometrically identical aggressor and victim lines in a 130nm technology is obtained using both the proposed metric and [14]. The line length varies from $4-8 \mathrm{~mm}$, and the supply voltage is $1.3 \mathrm{~V}$. To accurately model the interconnect, every $2 \mu \mathrm{m}$ of each line segment is modeled with an RC ladder network.

Table 3: Run time comparison between the proposed noise metric and [14] using a $130 \mathrm{~nm}$ technology and $V_{D D}=1.3 \mathrm{~V}$

\begin{tabular}{|c|c|c|}
\hline \multirow{2}{*}{$\begin{array}{c}\text { Wire-length } \\
(\mathbf{m m})\end{array}$} & \multicolumn{2}{|c|}{ Run-Time (sec) } \\
\cline { 2 - 3 } & Kuhlmann [14] & The proposed metric \\
\hline \hline 4 & 3 & 1 \\
\hline 5 & 3 & 1 \\
\hline 6 & 4 & 2 \\
\hline 7 & 4 & 3 \\
\hline 8 & 5 & 2 \\
\hline
\end{tabular}


Figures 9 (a), (b), and (c) show the crosstalk voltage waveforms obtained by using HSPICE simulation for the last three experiments in Table 1, where the aggressor and victim driver sizes are very different. As mentioned earlier in this section, it is assumed that the peak value of the crosstalk occurs approximately at $t=t_{r(f)}$. As the current drive capability of the line drivers decreases, the peak value of the crosstalk may occur further way from $t=t_{r(f)}$. As an example, Fig. 10 shows the HSPICE simulation result for the far-end crosstalk at the victim net along with the input waveform to the aggressor net. As seen in this figure, the time $t_{\max }$ approximated by [15] is quite different from $t_{r(f)}$. To examine the accuracy of the proposed metric for this case, Table 1 includes experiments where the source resistances $R_{s 1}$ and $R_{s 2}$ are large, and the neighboring lines are long. For instance, in one experiment given in Table 1, where the line length is $4 m m$, per-unit length parameters are $c_{1}=c_{2}=90 \mathrm{pF} / \mathrm{m}, r_{1}=r_{2}=11 \mathrm{k} \Omega / \mathrm{m}, c_{c}=120 \mathrm{pF} / \mathrm{m}$, and the source resistances are $R_{s 1}=160 \Omega$ and $R_{s 2}=160 \Omega$. Our metric predicts the peak crosstalk value to be $0.232 \mathrm{~V}$, which constitutes less than $5.94 \%$ estimation error compared to the HSPICE result. Notice that the error is kept below 5.94\% although the peak value does not occur at $t_{r}$ It is easily proved that the proposed noise analytical model will result in accurate noise amplitude-pulsewidth product. Therefore, we can state that our metric calculates the two important attributes of the capacitive crosstalk (i.e., the peak value and the noise pulse width) with a rather high accuracy.

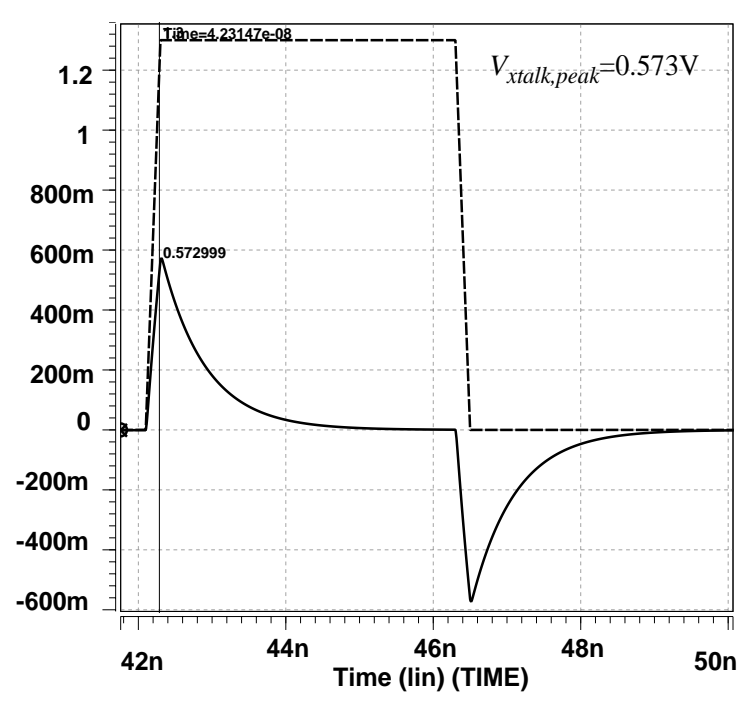

(a)

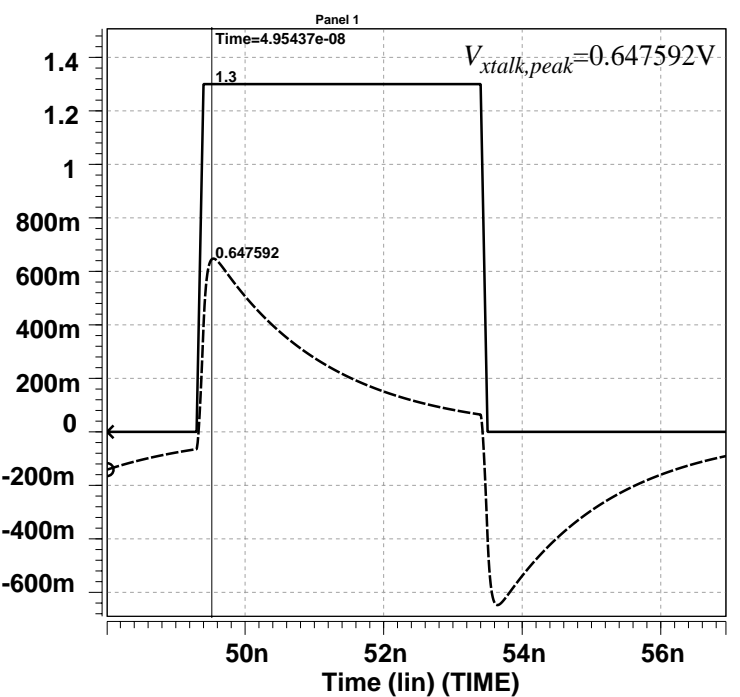

(b) 


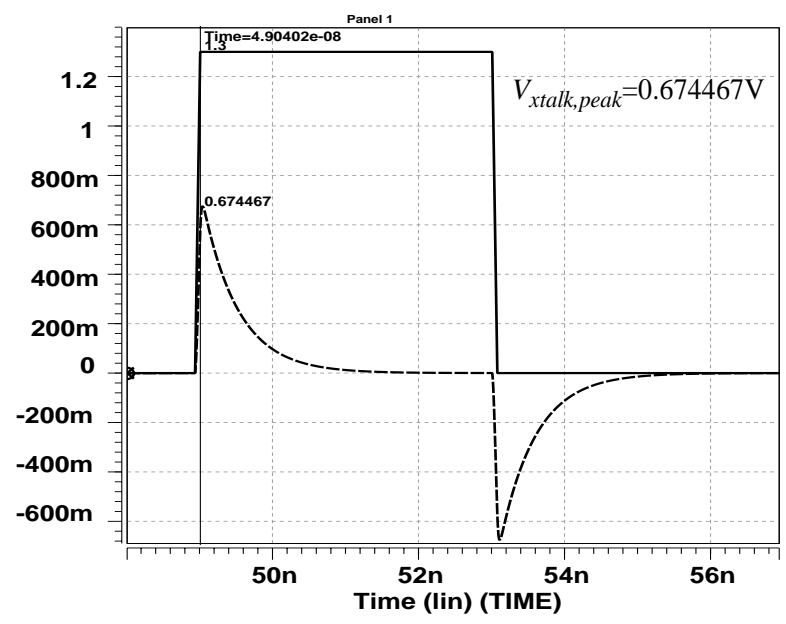

(c)

Fig. 9. Crosstalk waveforms obtained using HSPICE simulations for the last three experiments in Table 1.

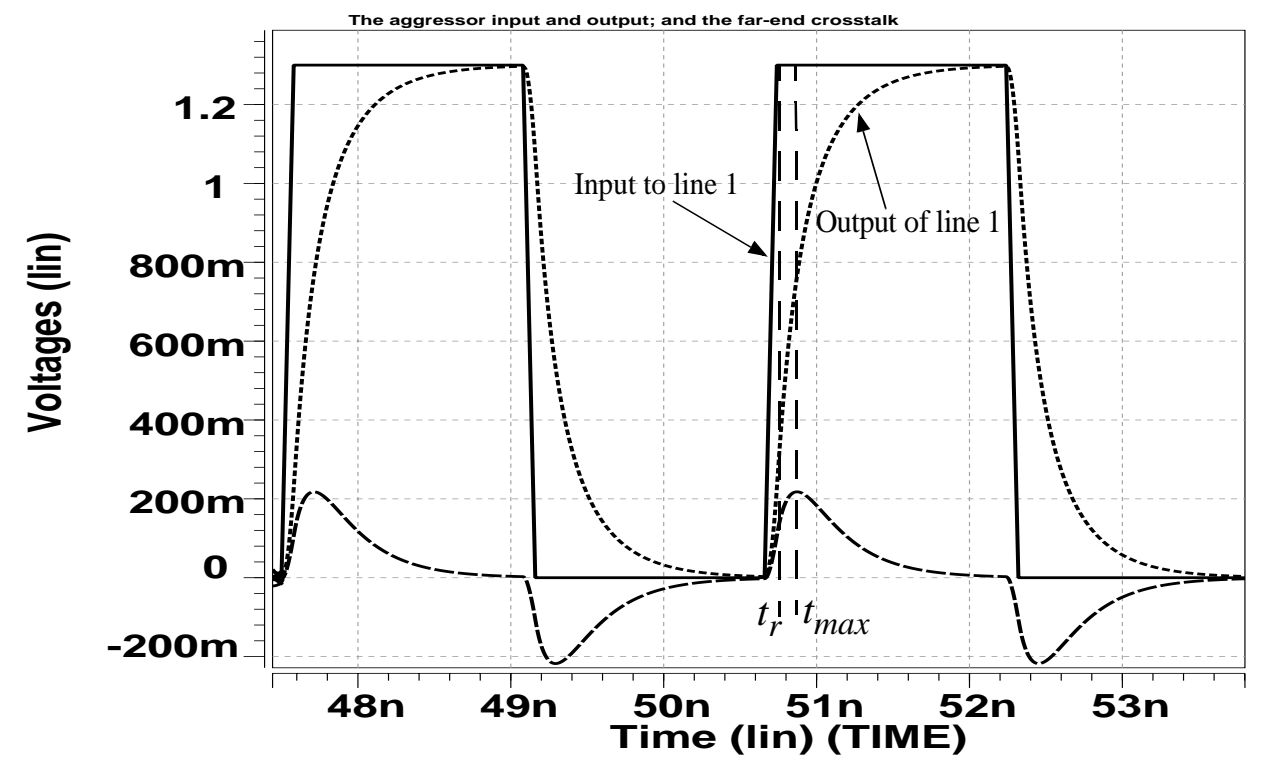

Fig. 10. The voltage waveforms at the input and output terminals of two adjacent interconnects of $4 m m$ length and with per-unit length electrical parameters $c_{1}=c_{2}=90 \mathrm{pF} / \mathrm{m}, r_{1}=r_{2}=11 \mathrm{k} \Omega / \mathrm{m}, c_{c}$ $=120 \mathrm{pF} / \mathrm{m}$.

The proposed analytical model can be extended to any RC tree network. Shown in Fig. 11 is the circuit consisting of a tree network a single interconnect line as a victim net. Such interconnect topology is often encountered in an integrated circuit, where local clock distribution networks are placed in the neighborhood of other signal lines. The circuit is realized in 130nm CMOS technology. 


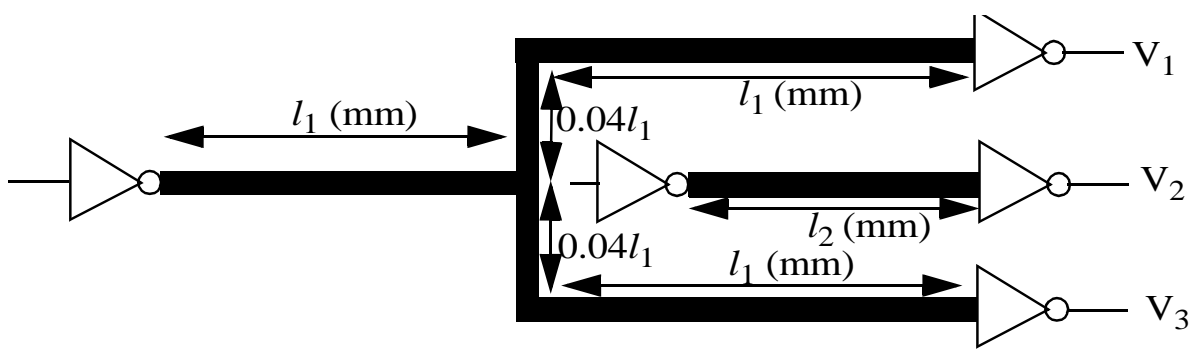

Fig. 11. A simple tree-network capacitively coupled to a single victim net.

Our model is compared with [10], [11], and [14]. Table 4 reports the results of these comparisons for a wide range of rise-times varying between 40ps and 400ps, different line lengths, and different driver sizes. Without loss of generality, we assume that $l_{2}=0.6 l_{1}$. To accurately model the interconnect, we model every $20 \mu \mathrm{m}$ of each line segment with an RC ladder network, which results in sufficiently accurate HSPICE simulation for $l_{1}$ varying

Table 4: The results of simulations on the crosstalk in the victim net of Fig. 12 using star-HSPICE, paper [10], paper [11], and our metric using a 130nm technology and $V_{D D}=1.3 \mathrm{~V}$

\begin{tabular}{|c|c|c|c|c|c|c|c|c|c|c|c|c|c|c|}
\hline $\begin{array}{l}\mathrm{c}_{1} \\
(\mathrm{pF} / \mathrm{m})\end{array}$ & $\begin{array}{l}\mathbf{r}_{1} \\
(\mathbf{k} \Omega / \mathbf{m})\end{array}$ & $\begin{array}{l}\mathbf{r}_{2} \\
(\mathbf{k} \Omega / \mathbf{m})\end{array}$ & $\begin{array}{l}\mathbf{c}_{2} \\
(\mathrm{pF} / \mathrm{m})\end{array}$ & $\begin{array}{l}\mathrm{c}_{\mathrm{c}} \\
(\mathrm{pF} / \mathrm{m})\end{array}$ & $\mathbf{R}_{\mathrm{s} 1}$ & $\mathbf{R}_{\mathrm{s} 2}$ & $\begin{array}{l}C_{\text {out1 }} \\
(\mathbf{p F})\end{array}$ & $\begin{array}{l}\mathrm{C}_{\text {out2 }} \\
(\mathrm{pF})\end{array}$ & $\begin{array}{l}\mathbf{t}_{\mathbf{r}} \\
\text { nsec }\end{array}$ & $\begin{array}{l}\mathrm{l}_{1} \\
(\mathrm{~mm})\end{array}$ & $\begin{array}{l}\text { HSPICE } \\
\text { volts }\end{array}$ & $\begin{array}{l}\text { Devgan } \\
\text { volts }\end{array}$ & $\begin{array}{l}\text { Vittal } \\
\text { volts }\end{array}$ & $\begin{array}{l}\text { Ours } \\
\text { volts }\end{array}$ \\
\hline 72 & 9.55 & 9.55 & 72 & 150 & 527 & 527 & 0.2 & 0.1 & 0.04 & 0.7 & 0.294 & 3.24 & 0.331 & $\overline{0.324}$ \\
\hline 92 & 9.3 & 10 & 80 & 170 & 270 & 400 & 0.06 & 0.2 & 0.06 & 2.5 & 0.469 & 4.5 & 0.511 & 0.502 \\
\hline 101 & 12 & 12 & 101 & 150 & 140 & 150 & 0.1 & 0.2 & 0.08 & 1.2 & 0.282 & 0.662 & 0.328 & 0.297 \\
\hline 130 & 13 & 20 & 100 & 220 & 670 & 720 & 0.3 & 0.05 & 0.09 & 1.6 & 0.368 & 4.36 & 0.425 & 0.401 \\
\hline 140 & 17 & 17 & 100 & 200 & 350 & 350 & 0.3 & 0.2 & 0.12 & 2 & 0.326 & 2.02 & 0.39 & 0.362 \\
\hline 140 & 17 & 17 & 100 & 200 & 30 & 30 & 0.07 & 0.08 & 0.06 & 2 & 0.46 & 0.85 & 0.531 & $\overline{\mathrm{0.488}}$ \\
\hline
\end{tabular}

Table 5: Error comparison for three noise metrics

\begin{tabular}{|c|c|c|c|}
\hline & $\begin{array}{l}\text { \% Error } \\
\text { Devgan's }\end{array}$ & $\begin{array}{l}\text { \%Error } \\
\text { Vittal's }\end{array}$ & $\begin{array}{c}\text { \% Error } \\
\text { Ours }\end{array}$ \\
\hline & 1000 & 12.6 & 10.2 \\
\hline & 1660.8 & 6.64 & 13.95 \\
\hline & 859.5 & 8.96 & 7.04 \\
\hline & 134.8 & 16.31 & 5.32 \\
\hline & 1888.4 & 16.52 & 14.2 \\
\hline & $\mathbf{0}$ & 7.63 & 0.85 \\
\hline & 1084.8 & 15.49 & 8.97 \\
\hline & 425 & 9.6 & 5.1 \\
\hline & 84.78 & 15.43 & 6.1 \\
\hline Average & 793.12 & 12.13 & 7.97 \\
\hline Maximum percentage error & 1888.4 & 16.52 & 14.2 \\
\hline
\end{tabular}


between $0.7 \mathrm{~mm}$ and $2 \mathrm{~mm}$ ( $c f$. Table 4 ). Table 5 shows the estimated error of each model as compared to HSPICE results.

The susceptibility of logic gates to noise depends not only on the peak amplitude of the crosstalk noise but also on its duration. For example, digital circuits can often tolerate (and indeed filter out) spike-like crosstalk noise with a large peak amplitude and very small noise pulse width [10]. In static CMOS logic circuits, the peak amplitude of crosstalk does not result in loss of signal values. Instead, it tends to cause an increase in propagation delay along the victim line, which in turn may cause setup time violation in high-speed circuits. These observations motivate the need for determining the noise pulse-width and the time-domain noise waveform.

Given the equivalent time constants of the crosstalk noise, $\tau_{d_{j}}^{(r)}$ and $\tau_{d_{j}}^{(f)}$, the peak amplitudes of the crosstalk noise, $\mathbf{V}_{2, S S}^{(r)}$ and $\mathbf{V}_{2, S S}^{(f)}$, corresponding to the rising and falling transitions of the input signal to the aggressor line, and the peak amplitudes of the crosstalk waveform, the noise waveform is readily calculated. For the general case of unequal rise and fall times, the noise waveform at each clock cycle time of $T_{c}$ is computed as follows:

$$
v_{2}(t)=v_{2 r}(t) u(t)+\left|v_{2 f}\left(t-\frac{T_{c}}{2}\right) u\left(t-\frac{T_{c}}{2}\right)\right|
$$

where $v_{2 r}(t) u(t)$ and $v_{2 f}(t) u(t)$ are defined as follows:

$$
v_{2 r}(t) u(t)= \begin{cases}\mathbf{V}_{2, s s}^{(r)}\left(\mathbf{I}-\exp \left(\operatorname{diag}\left(-\frac{t}{\tau_{d_{j}}^{(r)}}\right)\right)\right) & 0 \leq t \leq t_{r} \\ \mathbf{V}_{2, \text { max }}^{(r)} \exp \left(\operatorname{diag}\left(-\frac{t-t_{r}}{\tau_{d_{j}}^{(r)}}\right)\right) & t \geq t_{r}\end{cases}
$$




$$
v_{2 f}(t) u(t)=\left\{\begin{array}{ll}
\mathbf{V}_{2, s s}^{(f)}\left(\mathbf{I}-\exp \left(\operatorname{diag}\left(-\frac{t}{\tau_{d_{j}}^{(f)}}\right)\right)\right) & 0 \leq t \leq t_{f} \\
\mathbf{V}_{2, \text { max }}^{(f)} \exp \left(\operatorname{diag}\left(-\frac{t-t_{f}}{\tau_{d_{j}}^{(f)}}\right)\right) & t \geq t_{f}
\end{array} \quad \text { for } j=1,2, \ldots, N\right.
$$

Notice that having the noise waveform gives us the maximum amount of information regarding the noise behavior including the peak amplitude of crosstalk noise and the noise pulse width. This information allows designers to find better solutions for noise avoidance. Fig. 12 compares Eq. (12) with HSPICE simulation for a pair of capacitively coupled nets. As one can see, our new metric accurately predicts not only the noise peak amplitude but also the noise pulse width. Indeed, the effective pulse width is estimated with a maximum error of only $5 \%$. Our metric can easily be applied to the general case of having several parallel runs of on-chip interconnects (on the same metal layer or on different metal layers) by using the superposition principle [19].

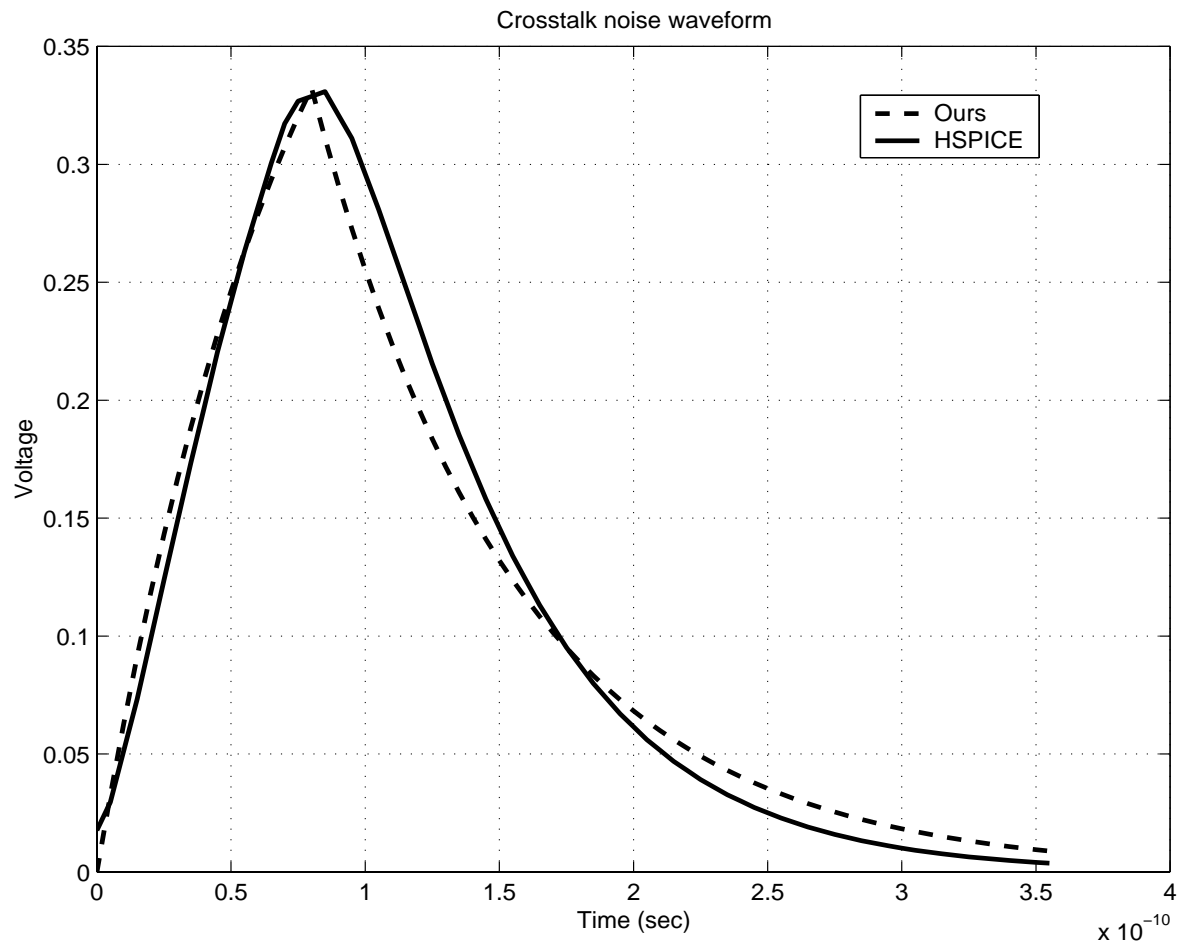

Fig. 12. Crosstalk noise waveforms for two coupled transmission lines. 
Fig. 13 shows the change in crosstalk when the input rise time varies from 50ps to $300 \mathrm{ps}$ while all of the geometrical parameters are fixed.

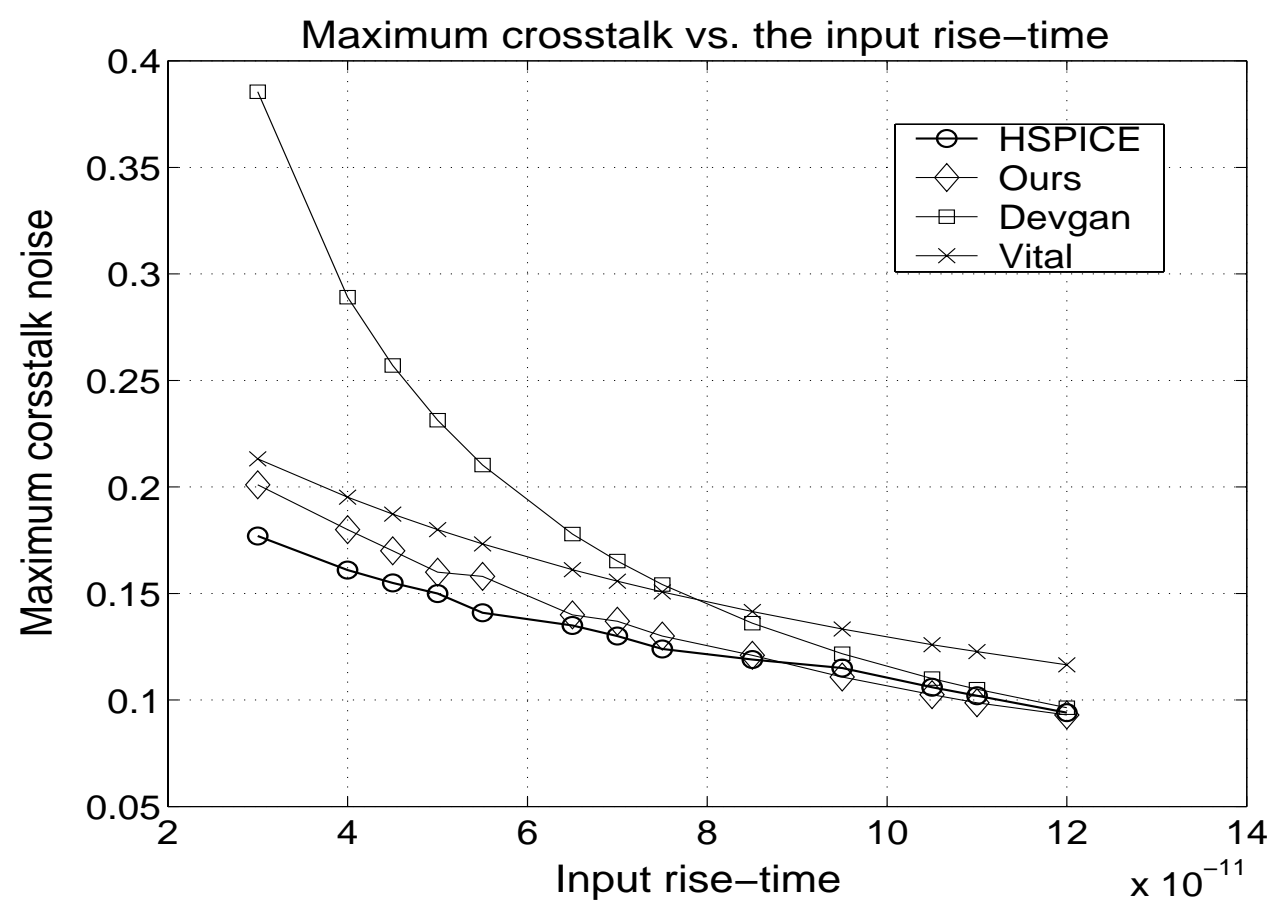

Fig. 13. Maximum crosstalk noise vs. input rise-time.

Comparing HSPICE results with our metric confirms that one achieves a high accuracy with our noise metric over a wide range of input rise-times. As expected, for long rise-times, Devgan's metric accurately predicts the peak amplitude of the noise. Vittal's metric exhibits higher fidelity compared to Devgan's, i.e., its estimation error remains roughly constant and does not present as large a dynamic range as Devgan's. Recall that, in [10], the authors use geometric considerations to obtain an expression for the crosstalk noise. As a result, they do not account for effects of the non-ideal delays on the crosstalk peak estimation. In contrast, our metric is based on the actual characteristics of capacitively coupled RC circuits that are derived from simulations. In summary, our metric is more accurate than those proposed in [10] and [11].

\subsection{Crosstalk noise estimation for the saturating exponential input}


The single-pole model proposed in Section 2.2 can easily be extended to predict the corsstalk noise when the input to the agressor line is a saturating exponential signal as explained next. Consider

$$
V_{s}(t)=V_{D D}\left(1-e^{-t / \tau_{s}}\right)
$$

where $\tau_{s}$ is the input time-constant. The crosstalk noise at the victim nodes becomes:

$$
\begin{array}{r}
v_{2}(t)= \\
V_{D D}\left[\mathbf{I}-\operatorname{diag}\left(\frac{1}{\tau_{d_{j}}^{(e x)}-\tau_{s}}\right)\left[\operatorname{diag}\left(\frac{1}{\tau_{d_{j}}^{(e x)}}\right) \exp \left(\operatorname{diag}\left(-\frac{t}{\tau_{d_{j}}^{(e x)}}\right)\right)-\operatorname{diag}\left(\frac{1}{\tau_{s}}\right) \exp \left(\operatorname{diag}\left(-\frac{t}{\tau_{s}}\right)\right)\right]\right] \\
\quad \text { for } j=1,2, \ldots, N
\end{array}
$$

where $\tau_{d_{j}}^{(e x)}$ represents the time constant of the $j$-th node in the victim net under an exponential input excitation. $\tau_{d_{j}}^{(e x)}$ is roughly equal to $\tau_{d_{j}}^{(e x)}=\left(\tau_{s} \ln 2 / t_{r}\right) \tau_{d_{j}}$ where $t_{r}$ is the risetime of the curve-fitting flattened ramp input, and $\tau_{d j}$ is the the time constant of the $j$-th node in the victim net under the curve-fitting flattened ramp input. In fact, this approximation contributes to a larger noise estimation error.

Tables 6 and 7 demonstrates the accuracy of the proposed metric in calculating the peak amplitrude of the coupling noise of two capacitively coupled interconnects in the presence of a saturated expontential signal at the input of the aggressor net. The noise estimation of the proposed metric is compared with the HSPICE simulation, and the techniques presented in [10], [12], and [14]. All comparisons are made using the device parameters for a 130nm standard CMOS process. The coupled lengths of the adjacent interconnects are varied from 1.1-8mm. 
Table 6: The results of simulations on the two capacitively coupled transmission lines using star-HSPICE, comparing methods of references [10], [13], [14] and our proposed metric using a 130nm CMOS technology and $V_{D D}=1.3 \mathrm{~V}$.

The aggressor line input is a saturated exponential signal.

\begin{tabular}{|c|c|c|c|c|c|c|c|c|c|c|c|c|c|c|c|}
\hline $\begin{array}{l}\mathrm{c}_{1} \\
(\mathrm{pF} / \mathrm{m})\end{array}$ & $\begin{array}{l}\mathbf{r}_{1} \\
(\mathbf{k} \Omega / \mathbf{m})\end{array}$ & $\begin{array}{l}\mathbf{r}_{2} \\
(\mathbf{k} \Omega / \mathbf{m})\end{array}$ & $\begin{array}{l}\mathrm{c}_{2} \\
(\mathrm{pF} / \mathrm{m})\end{array}$ & $\begin{array}{l}\mathrm{c}_{\mathrm{c}} \\
(\mathrm{pF} / \mathrm{m})\end{array}$ & $\mathbf{R}_{\mathrm{s} 1}$ & $\mathbf{R}_{\mathrm{s} 2}$ & $\begin{array}{l}\mathrm{C}_{\text {out1 }} \\
(\mathrm{pF})\end{array}$ & $\begin{array}{l}\mathrm{C}_{\text {out2 }} \\
(\mathrm{pF})\end{array}$ & $\begin{array}{l}\tau_{s} \\
\text { nsec }\end{array}$ & $\begin{array}{l}\mathbf{L} \\
(\mathbf{m m})\end{array}$ & $\begin{array}{l}\text { HSPICE } \\
\text { volts }\end{array}$ & $\begin{array}{l}\text { Pan } \\
\text { volts }\end{array}$ & $\begin{array}{l}\text { Vittal } \\
\text { volts }\end{array}$ & $\begin{array}{l}\text { Kuhlmann } \\
\text { volts }\end{array}$ & $\begin{array}{l}\text { Ours } \\
\text { volts }\end{array}$ \\
\hline 120 & 10 & 10 & 120 & 132 & $2 \mathrm{k}$ & $4 k$ & 0.06 & 0.07 & 0.14 & 1.1 & 0.204 & 0.272 & 0.281 & 0.224 & 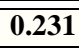 \\
\hline 108 & 15 & 15 & 108 & 200 & 20 & 30 & 0.2 & 0.1 & 0.16 & 1.3 & 0.086 & 0.091 & 0.103 & 0.09 & 0.087 \\
\hline 130 & 13 & 20 & 100 & 220 & 670 & 720 & 0.3 & 0.05 & 0.1 & 1.6 & 0.23 & 0.28 & 0.297 & 0.262 & 0.256 \\
\hline 140 & 17 & 17 & 100 & 200 & 350 & 350 & 0.3 & 0.2 & 0.14 & 2 & $\overline{0.198}$ & 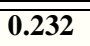 & 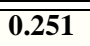 & 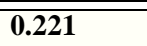 & $\begin{array}{c}0.22 \\
\end{array}$ \\
\hline 140 & 17 & 17 & 100 & 200 & 20 & 30 & 0.07 & 0.08 & 0.08 & 2 & 0.227 & 0.253 & $\begin{array}{l}0.268 \\
\end{array}$ & 0.243 & $\begin{array}{c}0.25 \\
\end{array}$ \\
\hline 90 & 11 & 11 & 90 & 120 & 160 & 160 & 0.3 & 0.2 & 0.1 & 4 & 0.201 & 0.242 & 0.251 & 0.222 & \begin{tabular}{c|}
0.21 \\
\end{tabular} \\
\hline 85 & 12 & 8.5 & 75 & 140 & 190 & 100 & 0.8 & 0.2 & 0.12 & 5 & $\mathbf{0 . 1 2 7}$ & 0.143 & 0.162 & 0.141 & 0.138 \\
\hline 65 & 13 & 7 & 120 & 170 & 600 & 80 & 0.08 & 0.5 & 0.06 & 6 & 0.072 & $\mathbf{0 . 0 8 3}$ & 0.089 & 0.074 & $\begin{array}{c}0.079 \\
\end{array}$ \\
\hline 110 & 6.5 & 7 & 90 & 180 & 80 & 75 & 0.6 & 0.7 & 0.1 & 7 & 0.218 & 0.241 & 0.256 & 0.232 & 0.239 \\
\hline 100 & 8 & 14 & 40 & 110 & 200 & $1 \mathrm{k}$ & 0.8 & 0.05 & 0.08 & 8 & $\overline{0.441}$ & $\overline{0.492}$ & $\overline{0.534}$ & $\overline{0.472}$ & $\overline{\overline{0.461}}$ \\
\hline
\end{tabular}

Table 7: Percentage Error comparison for methods of references [10], [13], [14] and our proposed metric

\begin{tabular}{|c|c|c|c|c|}
\hline & $\begin{array}{c}\text { \% Error } \\
\text { Pan's }\end{array}$ & $\begin{array}{l}\% \text { Error } \\
\text { Vittal's }\end{array}$ & $\begin{array}{c}\text { \%Error } \\
\text { Kuhlmann }\end{array}$ & $\begin{array}{c}\text { \% Error } \\
\text { Ours }\end{array}$ \\
\hline & 33.1 & 37.75 & 9.8 & 13.24 \\
\hline & 5.81 & 19.77 & 4.65 & 1.16 \\
\hline & 21.7 & 29.13 & 13.91 & 11.3 \\
\hline & 17.2 & 26.77 & 11.6 & 11.1 \\
\hline & 11.45 & 18.06 & 7.05 & 10.13 \\
\hline & 20.4 & 24.88 & 10.45 & 4.48 \\
\hline & 12.6 & 27.56 & 11.02 & 8.66 \\
\hline & 15.28 & 23.61 & 2.78 & 9.72 \\
\hline & 10.55 & 17.43 & 6.42 & 9.63 \\
\hline & 11.56 & 21.09 & 7.03 & 4.54 \\
\hline Average & 15.97 & 24.6 & 8.47 & 8.4 \\
\hline Maximum percentage error & 33.1 & 37.75 & 13.91 & 13.24 \\
\hline
\end{tabular}

\section{Conclusions}

In this paper, we presented an efficient analysis technique for the capacitive crosstalk noise calculation in sub-quarter micron VLSI interconnects. We derived closed-form expressions for the peak amplitude, the pulse width, and the time-domain waveform of crosstalk noise. Experimental results show that the maximum error is less than $13 \%$ and the average error is $5.82 \%$. 


\section{References}

[1] L. T. Pillage, R. A. Rohrer, "Asymptotic Waveform Evaluation for Timing Analysis", IEEE Trans. CAD, vol. 9, No. 4, pp. 352-366, April 1990.

[2] K. J. Kerns, A. T. Yang, "Stable and Efficient Reduction of Large, Multiport RC Networks by Pole Analysis via Congruence Transformations," IEEE Trans. CAD, vol. 16, pp. 734-744, 1997.

[3] P. Feldmann, R. W. Freund, "Efficient Linear Circuit Analysis by Pade Approximation via the Lanczos Process," IEEE Trans. CAD, vol. 14, pp. 639-649, May 1995.

[4] A. Odabasioglu, M. Celik, L. T. Pileggi, "PRIMA: Passive Reduced-Order Interconnect Macromodeling Algorithm," IEEE Trans. CAD, vol. 17, No. 8, pp. 645-654, Aug. 1998.

[5] P. Heydari, M. Pedram. "Balanced Truncation with Spectral Shaping for RLC Interconnects," IEEE Proc. ASP-DAC, pp. 203-208, Jan. 2001.

[6] P. Heydari, M. Pedram, "Model Reduction of Variable Geometry Interconnects Using Variational Spectrally-Weighted Balanced Truncation," Proc. IEEE/ACM Int. Conf. Computer-Aided Design, San Jose, CA, Nov. 2001.

[7] K. Shepard, V. Narayanan, "Noise in Submicron Digital Design," Proc. IEEE/ACM Int. Conf. Computer-Aided Design, pp. 524-531, Nov. 1996.

[8] K. Shepard, V. Narayanan, P. C. Elmendorf, and G. Zheng, "GlobalHarmony: Coupled Noise Analysis for Full-Chip RC Interconnect Networks," Proc. IEEE/ACM Int. Conf. Computer-Aided Design, pp. 139-146, Nov. 1997.

[9] A. Vittal, M. Marek-Sadowska, "Crosstalk Reduction for VLSI," IEEE. Trans. Computer-Aided Design, vol. 16, pp. 290-298, Mar. 1997.

[10] A. Vittal, L. H. Chen, M. Marek-Sadowska, K. P. Wang, S. Yang "Crosstalk in VLSI Interconnections," IEEE. Trans. Computer-Aided Design, vol. 18, no. 12, pp. 1817-1824, 1999.

[11] A. Devgan, "Efficient Coupled Noise Estimation for On-chip Interconnects," IEEE Proc. ICCAD, pp. 147-153. Nov. 1997.

[12] M. Kuhlmann, S. S. Sapatnekar, K. K. Parhi, "Efficient Crosstalk Estimation," IEEE 
IEEE Int. Conf. Computer Design, , pp. 266-272, Oct. 1999.

[13] J. Cong, D,. Z. Pan, P. V. Srinivas, “Improved Crosstalk Modeling for Noise Constrained Interconnect Optimization,” IEEE Proc. ASP-DAC, pp. 373-378, Feb. 2001.

[14] M. Kuhlmann, S. S. Sapatnekar, "Exact and Efficient Crosstalk Estimation," IEEE Trans. Computer-Aided Design, vol. 20, no.7, pp. 858-866, July 2001.

[15] M. Takahashi, M. Hashimoto, H. Onodera, "Crosstalk Noise Estimation for Generic RC Trees,” IEEE Int. Conf. Computer Design, pp. 110-116, Sept. 2001.

[16] Li Ding, D. Blaauw, P. Mazumder, "Efficient Crosstalk Noise Modeling Using Aggressor and Tree Reductions," IEEE/ACM Int'l Conf. Computer-Aided Design, pp. 595-600, Nov. 2002.

[17] P. Heydari, M. Pedram, "Analysis and Reduction of Capacitive Coupling Noise in High-speed VLSI Circuits," IEEE Int'l Conf. on Computer Design (ICCD), pp. 104-109, Sept. 2001.

[18] T. H. Lee, The Design of CMOS Radio-Frequency Integrated Circuits, Cambridge University Press, pp. 146-152, 1998.

[19] Chua, L. O., Desoer, C. A., Kuh, E. S., Linear and Nonlinear Circuits, McGraw Hill, New York, 1987. 